\title{
Differential Renormalization of van der Waals Spin Models
}

\author{
Raf Dekeyser $^{1}$ and Attilio Stella ${ }^{1,2}$
}

\author{
Received July 12, 1979
}

An exact renormalization group equation in differential form is derived for spin systems with general many-body interactions in the van der Waals limit. This equation is solved and the free energy is determined as an integral along the renormalization trajectory in the interaction space. It is shown that the transformation can always be modified in such a way that an undetermined integration constant for the free energy vanishes exactly, also below the critical temperature. We also demonstrate how the invariance of the free energy under a parameterdependent equivalence transformation can provide information about the critical behavior of the system. In this alternative approach "dangerous" irrelevant variables play an essential role.

KEY WORDS: van der Waals spin systems; molecular field; differential renormalization; integral representation of free energy.

\section{INTRODUCTION}

The renormalization group approach to critical phenomena, whether formulated in Fourier space ${ }^{(1)}$ or in real space, ${ }^{(2)}$ is always connected with a reduction of the degrees of freedom of the system and a consequent rescaling of lengths. In the limit where the strength and inverse range of interaction simultaneously go to zero, systems with weak, long-range forces become equivalent to so called Weiss or van der Waals models, ${ }^{(3)}$ for which the notions of "length" and even dimensionality lose their usual meaning.

This rather singular situation, whose primary physical consequence is the breakdown of hyperscaling, requires a nonstandard renormalization group analysis. Such an analysis has been performed on the van der Waals spin system by Knops et al. ${ }^{(4)}$ with a discrete block transformation. In order to

\footnotetext{
${ }^{1}$ Instituut voor Theoretische Fysica, Katholieke Universiteit Leuven, Leuven, Belgium.

${ }^{2}$ On leave of absence from Istituto di Fisica e Unità GNSM del CNR, Università di Padova, Padova, Italy.
} 
discuss the peculiar properties of the model, they had to solve the apparent inconsistency of transformation-dependent exponents by showing the presence of "dangerous" irrelevant variables. ${ }^{3}$

Even if it is exact, the renormalization group method used in Ref. 4 is rather complicated, which contrasts, in some sense, with the relative simplicity of the model itself. What is more, their method suffers from a strong limitation, since they showed that below criticality their transformation could not be iterated indefinitely, not as one would expect in any renormalization group analysis. As a consequence, below criticality the free energy could not be computed by their renormalization method.

Recently a differential form of renormalization in real space has been proposed by Hilhorst et al. ${ }^{(6)}$ for the two-dimensional nearest-neighbor triangular Ising model. Some exact results were obtained by this method, ${ }^{(6,7)}$ which has also been applied to a few other, simpler solvable models. ${ }^{\left({ }^{8,9)}\right.}$ Flow equations in differential form are most suitable for discussing formal properties of the renormalization group. Even if equally exact, they are expected to be more powerful than the discrete transformations, particularly as far as the calculation of the free energy as an integral over the renormalization trajectory is concerned. A serious complication in the above differential approaches, however, is that one must introduce spatial inhomogeneities and anisotropies which complicate the flow equations in interaction space. This difficulty has made it impossible up to now to obtain general solutions for the renormalization trajectories, and consequently to compute the free energy, except in the case of the one-dimensional Ising model. ${ }^{(9)}$

In this work, we present an exact differential renormalization approach to the van der Waals spin system, which does not suffer from the limitations mentioned above in connection with Ref. 4. On the contrary, certain special features of the system - namely, the absence of length scale and dimensionality - make it rather amenable to a differential renormalization treatment, without the introduction of inhomogeneities and other similar complications. We are thus able to solve the flow equation in interaction space and to compute explicitly the free energy. This is also possible below criticality and in the presence of symmetry-breaking fields.

We believe that the scheme worked out and completely solved here represents an interesting realization of the differential renormalization technique, even though the features connected with length scale and dimensionality are evidently not present in our example.

The plan of the paper is as follows: In Section 2 we explicitly describe the class of models considered, and we derive the flow equations in the simplest form. In Section 3 we solve this equation, and we determine

\footnotetext{
${ }^{3}$ An alternative approach, based on the limit theorems of probability theory, has recently been proposed for mean field models by Ellis and Newman. ${ }^{(5)}$
} 
the free energy of the model as a trajectory integral along the flow lines. Section 4 is devoted to the pair-interaction model, and to the discussion of some problems encountered in the calculations when the initial Hamiltonian has certain features of symmetry breaking and metastable states. These problems are further and more generally analyzed in Section 5. It is shown that an arbitrariness in the definition of the transformation leads to different integral expressions for the free energy. The additional integration constant is shown to become zero for a certain set of normalizations for the transformation. In Section 6, a modified transformation is defined, which changes the behavior of the flow trajectories around the fixed point; the connection with the above-mentioned dangerous variables ${ }^{(4)}$ is indicated. The final section is devoted to concluding remarks; we also mention a difficulty and we suggest some possible extensions of the approach.

\section{DESCRIPTION OF THE MODEL AND THE INFINITESIMAL TRANSFORMATION}

The model that we want to study consists of a set of $N$ "spin" variables $S_{j}= \pm 1(j=1,2, \ldots, N)$. These spins interact via many-body potentials, which are invariant for the full permutation group of the $N$ indices. In the thermodynamic limit, these potentials are scaled down to zero by appropriate powers of $N$, such as to make the energy a truly extensive quantity. As mentioned in the introduction, the notions of distance and spatial dimension become meaningless with interactions of this type.

The Hamiltonian $H$ of such a mean-field-type model can be written as

$$
-\beta H(\{S\})=N e(m)
$$

where $\{S\}$ indicates a particular configuration, $\beta=1 / k_{\mathrm{B}} T$, and

$$
m=N^{-1} \sum_{j=1}^{N} S_{j}
$$

is the average magnetization, which can be treated as a continuous variable in the thermodynamic limit. We assume that $e(m)$ is a regular function in the interval $(-1,+1)$, and that $e(0)=0$.

The static thermodynamic properties of the system can be derived from the free energy per spin $F$, which is a functional of $e(m)$, defined by

$$
-\beta F=\lim _{N \rightarrow \infty} N^{-1} \ln Z_{N}=\lim _{N \rightarrow \infty} N^{-1} \ln \operatorname{Tr}_{\{S\}} \exp [N e(m)]
$$

$\operatorname{Tr}_{\{S\}}$ indicates the sum over all spin configurations and $Z_{N}$ is the partition function of the system.

One can easily compute $F$ exactly. Introducing the entropy function 


$$
S(m)=\ln 2-\frac{1}{2}[(1+m) \ln (1+m)+(1-m) \ln (1-m)]
$$

we can write

$$
\operatorname{Tr}_{\{S\}} \exp [N e(m)] \simeq \frac{N}{2} \int_{-1}^{+1} d m \exp \{N[e(m)+S(m)]\}
$$

asymptotically for large $N$. We thus obtain the result

$$
-\beta F=S(\bar{m})+e(\bar{m})
$$

where $\bar{m}$ maximizes the integrand of (5) and is then a solution of

$$
\dot{S}(m)+\dot{e}(m)=0
$$

where, to avoid notational confusion, we have used the dot to indicate differentiation with respect to $m$. To obtain in full generality the above simple result in a renormalization group context is not a trivial task.

The idea at the basis of the renormalization group approach is to compute the partition function of the system in successive steps, by gradually thinning out the degrees of freedom of the system. This basic process of thinning out leads to the definition of renormalized effective interactions between the remaining degrees of freedom in terms of the original interactions. This is called the renormalization transformation.

If the fraction of eliminated degrees of freedom in one such step remains finite in the thermodynamic limit, we have a discrete transformation for the interactions. If we are able to realize this reduction of degrees of freedom in such a way that the eliminated fraction tends to zero in the thermodynamic limit, then the renormalization equations acquire a differential character and we can properly speak of a differential flow in interaction space. That it is possible to establish these kinds of exact differential renormalization equations for any lattice system is not at all evident. On the contrary, one could suspect that this possibility is connected with the existence of a mapping of the star-triangle type for the model. ${ }^{(6)} \mathrm{Up}$ to now, indeed, these mappings, in combination with an ingenious use of inhomogeneities, anisotropies, and spatial domain boundary conditions, have been the only way through which one could consistently construct differential renormalization flows for realistic systems with short-range interactions. ${ }^{(6-9)}$ The van der Waals system which we have just described, however, allows a very easy and natural construction of the differential transformation without the necessity of startriangle relations.

The simplest way in which we can accomplish our task is to sum out one of the spin variables, e.g., $S_{N}$, in the partition function $Z_{N}$. This process can be interpreted as the construction of a system of $N-1$ degrees of freedom with new effective interactions. The fraction of eliminated degrees 
of freedom is $1 / N$, which goes to zero in the thermodynamic limit.

Let us define

$$
m^{\prime}=(N-1)^{-1} \sum_{j=1}^{N-1} S_{j}
$$

The trace over $S_{N}$ of the original Boltzmann factor depends only on $\mathrm{m}^{\prime}$, thus defining a new effective Boltzmann factor for the transformed system:

$$
\operatorname{Tr}_{S_{N}} \exp [N e(m)] \equiv \exp \left[(N-1) e^{\prime}\left(m^{\prime}\right)+g\right]
$$

By imposing, e.g., again $e^{\prime}(0)=0$, this equation allows us to determine both the transformed negative energy function $e^{\prime}$ and the constant term $g$.

The crucial point is now that, at least in the thermodynamic limit, the sum over $S_{N}$ in (9) can be performed exactly by expanding $e(m)$ around $m^{\prime}$. Omitting terms of order $N^{-2}$, which are not important in our analysis, we obtain

$$
g+(N-1) e^{\prime}\left(m^{\prime}\right)=N e\left(m^{\prime}\right)-m^{\prime} \dot{e}\left(m^{\prime}\right)+\ln \left[2 \cosh \dot{e}\left(m^{\prime}\right)\right]+O\left(N^{-1}\right)
$$

or

$$
g=\ln 2+\ln \cosh \dot{e}(0)
$$

and

$$
e^{\prime}(m)-e(m)=N^{-1}\left[e(m)-m \dot{e}(m)+\ln \frac{\cosh \dot{e}(m)}{\cosh \dot{e}(0)}\right]
$$

If we define a parameter $s$ to keep track of our decimation rate by

$$
\Delta s=-\Delta N / N=1 / N
$$

then the difference equation (12) transforms in the thermodynamic limit into a differential equation for the function $e(m, s)$, the negative energy function depending on the flow parameter $s$ :

$$
\frac{\partial e}{\partial s}=e-m \dot{e}+\ln \frac{\cosh \dot{e}(m, s)}{\cosh \dot{e}(0, s)}
$$

Since we normalize $e(0, s)=0$ for all $s$, we may as well try to solve first for $\dot{e}(m, s)$, which obeys the equation

$$
\frac{\partial \dot{e}}{\partial s}+(m-\tanh \dot{e}) \frac{\partial \dot{e}}{\partial m}=0
$$

This is a quasilinear partial differential equation ${ }^{(10)}$ in the variables $m$ and $s$. For the interactions of the Ising model on the triangular lattice, Hilhorst et $a{ }^{(6)}{ }^{(6)}$ obtained a system of equations of the same type, but with the spatial coordinates playing in some sense the role of our magnetization $m$. 
A nontrivial, regular fixed-point solution of (15) is given by

$$
\dot{e}^{*}=\tanh ^{-1} m
$$

which is the same fixed point as found in Ref. 6. By integrating this solution, we find that

$$
e^{*}(m)=\ln 2-S(m)
$$

where $S(m)$ is the entropy function (4). If we linearize the transformation around this fixed point by assuming

we find

$$
\dot{e}=\dot{e}^{*}+\psi(m)
$$

$$
\partial \psi / \partial s=\psi+O\left(\psi^{2}\right)
$$

This means that this fixed point is completely repulsive in all the directions in interaction space.

Because of the repulsive character of this fixed point, the standard analysis of the critical region in terms of the linearized renormalization equations breaks down for this model. This was already pointed out by Knops et al. ${ }^{(4)}$ These authors obtained the correct critical behavior of the system by taking into account the existence of equivalence transformations and dangerous variables. Although something analogous can be done for our differential transformation, as we will show in Section 6, it is more interesting to solve exactly the flow trajectories in the infinite-dimensional interaction space of the functions $\dot{e}(m)$. As we will see in the next section, it is then possible to obtain and compute an expression for the free energy $F$ as an integral along these trajectories, thus deriving all the properties of the system, including the critical ones. In this analysis, the nonlinear effects of the flow play a crucial role, and these effects are usually disregarded in the standard renormalization equation analysis. ${ }^{(1,2)}$

\section{SOLUTION OF THE DIFFERENTIAL EQUATION AND CALCULATION OF THE FREE ENERGY}

In order to solve the flow equation (15), we may use the standard method of the characteristic curves. ${ }^{(10)}$ These are given in parametric form by

$$
s=a+\lambda, \quad m=b e^{\lambda}+\tanh \dot{e}, \quad \dot{e}=c
$$

where $a, b$, and $c$ are integration constants and $\lambda$ is the curve parameter. The integration constants must be adjusted to the initial conditions. If, at $s=0$, i.e., before we start renormalizing, we have

$$
\dot{e}(m, s=0) \equiv \phi(m)
$$


then by elimination of the integration constants in (20), we obtain the solution of (15) in implicit form by

$$
\dot{e}(m, s)=\phi\left[m e^{-s}+\left(1-e^{-s}\right) \tanh \dot{e}(m, s)\right]
$$

The free energy corresponding to the Hamiltonian (1) is a functional of the energy function $e(m)$. It is thus natural to introduce an $s$-dependent function $F(s)$, which is the free energy corresponding to the energy function $e(m, s)$ :

$$
F(s) \equiv F(e(m, s))
$$

Our main interest of course lies in $F(0)$, the free energy corresponding to the original $e(m)=e(m, 0)$.

By forming the trace over the $N-1$ spin variables in (9), we obtain

$$
-\beta N F(e)=-\beta(N-1) F\left(e^{\prime}\right)+g
$$

or, in differential form,

$$
\partial(\beta F) / \partial s=\beta F+g
$$

The general solution of this equation is given by

$$
\beta F(s)=e^{s}\left\{C-\int_{s}^{\infty} d \sigma e^{-\sigma} g[e(\sigma)]\right\}
$$

Even when the integral on the right-hand side can be evaluated exactly, there is no direct way to obtain information on the constant $C$ from the renormalization equations. It has been argued that analogous terms, appearing both with discrete ${ }^{(11)}$ and with continuous ${ }^{(12)}$ transformations, may be neglected when there is no ordering field or spontaneous magnetization present. It is clear from (25) that $C$ must be zero when the limit of $e^{-s} F(s)$ is zero for $s$ going to infinity. The existence of this limit may be questionable whenever the renormalization trajectory flows away toward infinity in the interaction space. For the rest of this section, we will simply assume $C$ to be zero and concentrate on the integral (25). We postpone to the next two sections the discussion of whether the results obtained under this assumption correspond to the true free energy of the system.

With $C=0$, the free energy of Hamiltonian (1) is given by

$$
-\beta F=\int_{0}^{\infty} d s e^{-s} g[e(s)]=\ln 2+\int_{0}^{\infty} d s e^{-s} \ln \cosh \dot{e}(0, s)
$$

In order to obtain the thermodynamic properties of the system, it is thus sufficient to know $\dot{e}(m, s)$ for $m=0$ only. This quantity represents the effective magnetic field that has been built up by the renormalization scheme at the $s$ stage. One may thus expect that $\dot{e}(0, \infty)$ will represent the usual 
molecular field, which may be different from zero, even when the original field $h=\dot{e}(0,0)$ is zero. Equation (22), for $m=0$, describes this evolution of the molecular field, and we will study its behavior more explicitly with a specific example in the next section.

To end this section, we would like to present some of the previously obtained results in a more compact way. We define

$$
x=1-e^{-s}
$$

as the flow parameter, describing the fraction of degrees of freedom that have been eliminated; furthermore, we define

$$
h(x)=\dot{e}(0, s)
$$

as the effective magnetic field and

$$
Q(x)=\tanh h(x)
$$

According to (22), these quantities satisfy

$$
h(x)=\phi[x Q(x)]
$$

The free energy is obtained by partial integration of (26) as

$$
\begin{aligned}
-\beta F & =\ln 2+\ln \cosh h(1)-\int_{0}^{1} d x \times Q(x) \frac{d h}{d x} \\
& =\ln 2+\ln \cosh h(1)-\int_{0}^{1} d x \times Q\left(1-Q^{2}\right)^{-1} \frac{d Q}{d x}
\end{aligned}
$$

\section{EXAMPLE AND FURTHER ANALYSIS}

Let us test our results by applying them to the standard case, where the original Hamiltonian contains only pair interactions, apart from an external magnetic field:

$$
e(m, 0)=h_{0} m+\frac{1}{2} b m^{2}
$$

and thus

$$
\phi(m)=h_{0}+b m
$$

From (30) we see that $h(x)$ satisfies the equation

$$
h(x)=h_{0}+b x \tanh h(x)
$$

The free energy may be obtained from (31), in combination with the above formulas, as

$$
-\beta F=\ln 2+\ln \cosh h(1)-\frac{1}{2} b \tanh ^{2} h(1)
$$


The magnetization $\langle m\rangle$ can then be derived as

$$
\langle m\rangle=-\beta \partial F / \partial h_{0}=\tanh h(1)=\tanh \left(h_{0}+b\langle m\rangle\right)
$$

which is the usual mean-field equation.

It is well known that below criticality, i.e., for $b>1$, and for sufficiently small values of $h_{0}$ an equation like (36) formally also allows a metastable and an unstable solution. It should, however, be remembered that $h(x)$ is a continuous function of $x$. If we solve (34) graphically by looking at the intersection of the functions $f_{1}(h)=h-h_{0}$ and $f_{2}(h)=b x \tanh h$ for $b>1$ and for increasing values of $x$ (see Fig. 1), we find that only the stable solution of (36) can be attained in a continuous way when $x$ tends to unity. The condition of continuity for our renormalization trajectory thus selects in a unique way that solution of Eq. (36) which corresponds to the stable thermodynamic state. In Fig. 2 we sketch the behavior of $h(x)$ for $b>1$ and a positive $h_{0}$. It is clear that the continuity criterion forces us to disregard the disconnected part of the curve (dashed line). Above criticality, i.e., for $b<1$, the curve would have only one branch. When $h_{0}=0$, the solutions for $h(x)$ become symmetric about the $x$ axis (Fig. 3). The solid line in Fig. 3 should be considered as $h(x)$ in the limit $h_{0} \rightarrow+0$.

In this example it could thus be seen that with our renormalization approach we indeed obtain the correct results, even below the critical point and with an external magnetic field. This means that the assumption $C=0$ made in Eq. (25) was justified, contrary to what happened in other calculations. ${ }^{(11,12)}$ It is natural to ask how general is this result.

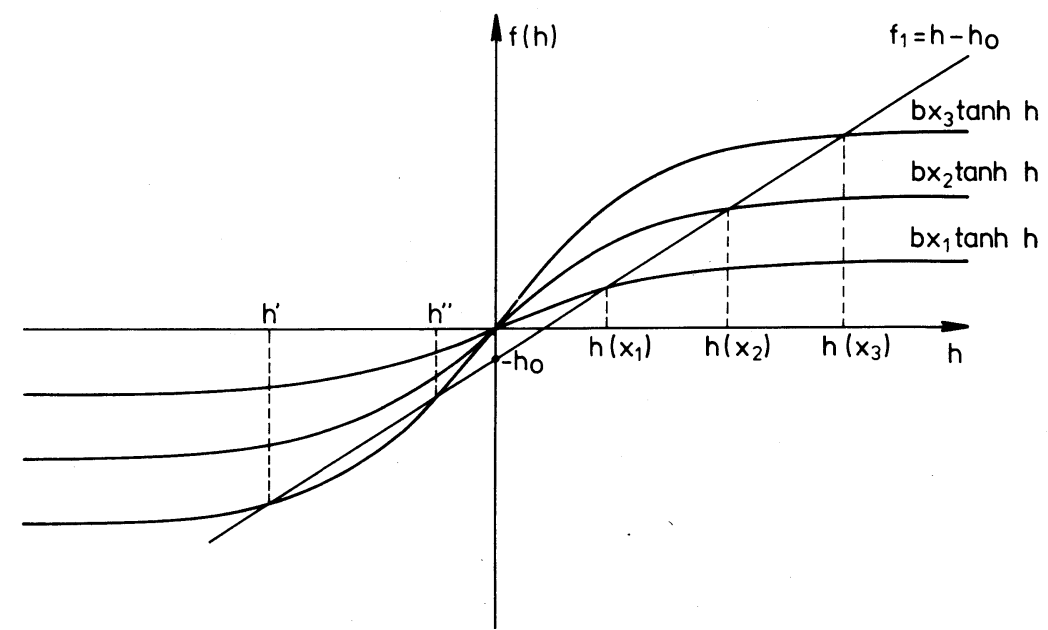

Fig. 1. Graphical solution of Eq. (34) for $x_{1}<x_{2}<x_{3}$. If $x_{3}=1, h^{\prime \prime}$ gives the unstable solution of the molecular field equation (36) and $h^{\prime}$ the metastable one. They do not belong to a continuous function $h(x)$ for $0<x<1$. 


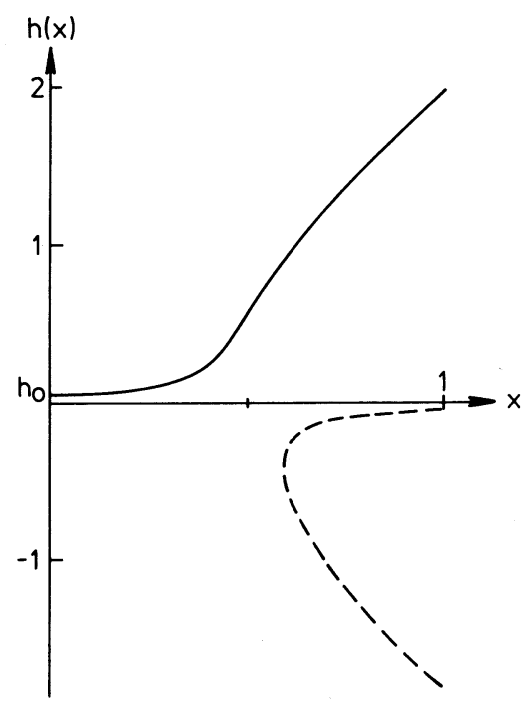

Fig. 2. Roots of Eq. (34) for the typical example $b=2, h_{0}=0.05$. Only the solid line corresponds to a continuous function for $0<x<1$.

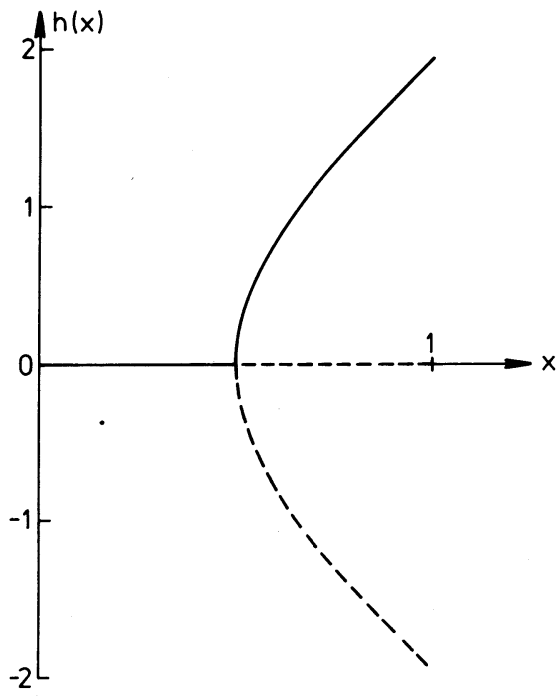

Fig. 3. Roots of Eq. (34) for $b=2$ and $h_{0}=0$. The solid line corresponds to the continuous solution in the limit $h_{0} \rightarrow 0+$. 
Let us therefore assume for the original Hamiltonian the general form

$$
e(m, 0)=e^{*}(m)+E(m)
$$

and

$$
\phi(m)=\dot{e}^{*}(m)+\psi(m)
$$

where, of course, $\psi(m)=\dot{E}(m)$. From (6) and (17) we see that the exact expression for the free energy is given in this case by

$$
-\beta F=\ln 2+E(\bar{m})
$$

where $\bar{m}$ represents the absolute maximum of $E(m)$. This implies that $\bar{m}$ satisfies

$$
\psi(\bar{m})=0
$$

but this is not sufficient to determine uniquely the correct $\bar{m}$, since all $m$ values that correspond to some extrema of $E(m)$ satisfy this equation. We must now compare our renormalization result (31) with this exact result (39).

Using our definitions (37)-(38) in (28)-(30), we have

$$
\tanh ^{-1} Q=\tanh ^{-1} x Q+\psi(x Q)
$$

For $x=1$, this means that $\psi[Q(1)]=0$, so $Q(1)$ satisfies Eq. (40), and it may eventually be identified with the magnetization $\bar{m}$, as in the pairinteraction example, with (36). If we take the derivative of (41) with respect to $x$, we see that

$$
\left(1-Q^{2}\right)^{-1} \frac{d Q}{d x}=\left[\left(1-x^{2} Q^{2}\right)^{-1}+\psi^{\prime}(x Q)\right] \frac{d(x Q)}{d x}
$$

and, using this in (31), we obtain

$$
-\beta F-\ln 2=-\int_{0}^{Q(1)} d y y \psi^{\prime}(y)=\int_{0}^{Q(1)} d y \psi(y)=E[Q(1)]
$$

This is the desired result (39).

We are left with the problem of determining whether the value of $Q(1)$, belonging to a continuous function $Q(x)$ for $0<x<1$, really corresponds to $\bar{m}$, the absolute maximum of $E(m)$, and not to some other extremum. It is easy to see that this is not always the case. Indeed, Eq. (41) can be solved graphically if we write it, e.g., under the form

$$
Q(1-x) /\left(1-x Q^{2}\right)=\tanh \psi(x Q)
$$

In order to avoid the problems with spontaneous symmetry breaking, let us assume that there is an initial magnetic field $h_{0}>0$ present, and let us take for $E(m)$ and $\psi(m)$ the form sketched in Figs. $4 \mathrm{a}$ and $4 \mathrm{~b}$. The left-hand 


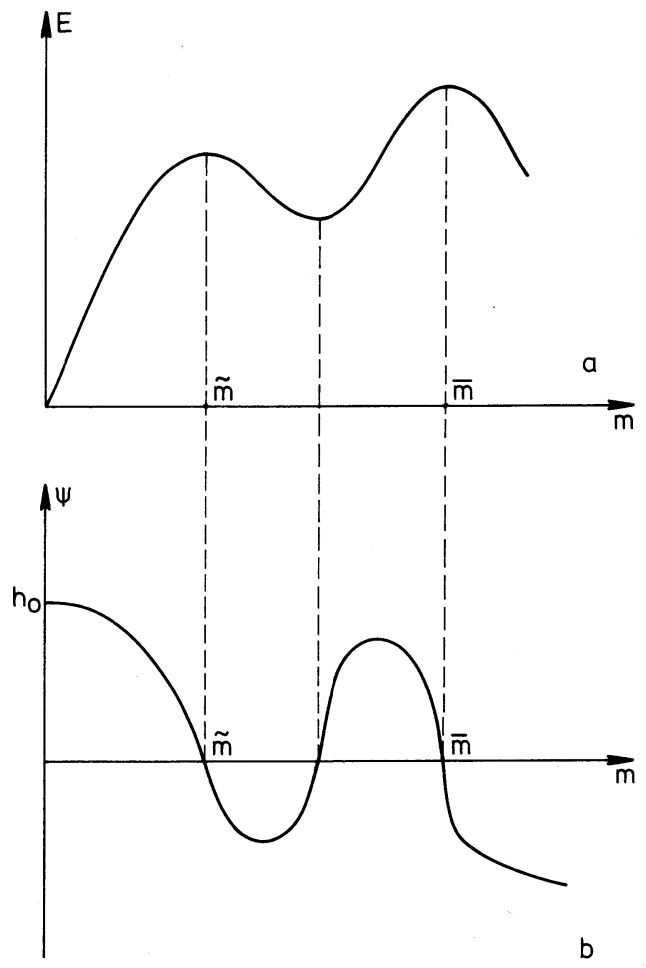

Fig. 4. Sketch of the behavior of (a) the function $E(m)$ and (b) $\psi(m)$, for which we do not obtain the correct value for the free energy if we assume $C=0$ in (25). The $Q(1)$ becomes equal to $\tilde{m}$ instead of $\bar{m}$.

side of (44) is always positive for $0<x, Q<1$, and the tanh in the righthand side preserves the sign of its argument. A simple graphical construction then shows that $Q(x)$ will tend to $\tilde{m}$, when $x$ goes to unity, and not to the value $\bar{m}$ that maximizes $E(m)$. It can actually be seen that in the general case $Q(1)$ will always be equal to the value of $m$ that corresponds to the first maximum encountered in the direction of the magnetization $h_{0}$.

In these special cases, where $Q(1)$ corresponds to an extremum, but not the absolute maximum of $E(m)$, our conclusion must be that the constant $C$ of $(25)$ is not zero, but

$$
C=E[Q(1)]-E(\bar{m})
$$

In the next section we will see that, within the above general renormalization scheme, it is always possible to find appropriate modifications of the process of resummation of the free energy, expressed by the basic equation 
(24), in such a way that this free energy is given by an integral expression as in (25), with the constant $C$ strictly equal to zero.

\section{EQUIVALENT EXPRESSIONS FOR THE FREE ENERGY}

When defining our transformation in Section 2, we had to impose $e^{\prime}(0)=e(0)(=0)$ in order to determine both $e^{\prime}(m)$ and $g$ from the single equation (9). This particular choice, however, is quite arbitrary; in other words, there is no unique way of identifying $e^{\prime}$ and $g$ from (9). We could, e.g., as well impose the condition

$$
e^{\prime}(M)=e(M)
$$

for some arbitrary $M(|M| \leqslant 1)$, and more generally,

$$
e(M, s) \equiv e(M) \quad \text { for all } s
$$

According to (10), this leads to

$$
g=g_{M}=\ln 2+\ln \cosh \dot{e}(M)-M \dot{e}(M)+e(M)
$$

One can easily check that the corresponding modification in the definition of $e^{\prime}$ does not change the differential equation (15) for $\dot{e}$, since the additional terms disappear when taking the derivative with respect to $M$. This means that the solutions (22) for the trajectories $\dot{e}(m, s)$ in the interaction space are unaltered, and that they do not need to bear a subscript $M$, as $g_{M}$. The basic difference with the previous section lies in the expression for the free energy, which can now be written as

$$
\begin{aligned}
-\beta F & =C(M)+\int_{0}^{\infty} d s e^{-s} g_{M} \\
& =C(M)+\ln 2+e(M)+\int_{0}^{\infty} d s e^{-s}[\ln \cosh \dot{e}(M, s)-M \dot{e}(M, s)]
\end{aligned}
$$

The question that we now want to investigate on the basis of this equation is how $C(M)$ behaves and, in particular, whether we can find some values of $M$ for which $C(M)=0$.

First of all, it is easy to show that $C(M)$ must be a step function. Indeed, if we assume that the derivative of $C(M)$ exists for a given value of $M$, then this derivative is zero. This can be shown by differentiating (48), where the free energy is of course constant with respect to $M$ :

$0=\dot{C}(M)+\dot{e}(M, 0)+\int_{0}^{\infty} d s e^{-s}\left\{[\tanh \dot{e}(M, s)-M] \frac{\partial \dot{e}}{\partial M}-\dot{e}(M, s)\right\}$ 
Using the differential equation (15), we can transform (49) to

$$
\dot{C}(M)+\dot{e}(M, 0)=-\int_{0}^{\infty} d s \frac{\partial}{\partial s}\left[\dot{e}(M, s) e^{-s}\right]=\dot{e}(M, 0)
$$

and thus

$$
\dot{C}(M)=0
$$

This equation holds almost everywhere, but not for those values of $M$ that are points of instability for the renormalization flow. For such values of $M$ it would not be legitimate to interchange the integral in (48) with the differentiation with respect to $M$.

After establishing this step-function character of $C(M)$, let us now determine the discontinuities in $C(M)$. Using again our definition (27) for $x$, we may write after separate partial integrations for each term in the integrand of $(48)$

$$
\begin{aligned}
-\beta F- & C(M)-\ln 2-e(M) \\
= & \int_{0}^{1} d x[\ln \cosh \dot{e}(M, x)-M \dot{e}(M, x)] \\
= & \ln \cosh \dot{e}(M, 1)-M \dot{e}(M, 0) \\
& \quad-\int_{0}^{1} d x[x \tanh \dot{e}(M, x)+M(1-x)] \frac{\partial \dot{e}(M, x)}{\partial x}
\end{aligned}
$$

Introducing now the definitions

$$
\begin{aligned}
& Q(x)=\tanh \dot{e}(M, x) \\
& Z(x)=x Q(x)+M(1-x)
\end{aligned}
$$

these quantities satisfy, from (22) and (38), the condition

$$
\tanh ^{-1} Q=\tanh ^{-1} Z+\psi(Z)
$$

Since $Z(1)=Q(1)$, it follows that

$$
\psi[Q(1)]=0
$$

or $Q(1)$ is again one of the values of $m$ that correspond to an extremum of $E(m)$. From (55) it follows also that

$$
\left(1-Q^{2}\right)^{-1} Q^{\prime}=\left[\left(1-Z^{2}\right)^{-1}+\psi^{\prime}(Z)\right] Z^{\prime}
$$


This may be substituted in the free energy calculation (52):

$$
\begin{aligned}
\beta F+ & C(M)+e(M)-M \dot{e}(M, 0)+\ln 2 \cosh \dot{e}(M, 1) \\
& =\int_{0}^{1} d x Z(x)\left(1-Q^{2}\right)^{-1} Q^{\prime}=\int_{0}^{1} d x Z\left[\left(1-Z^{2}\right)^{-1}+\psi^{\prime}(Z)\right] Z^{\prime} \\
& =\left.\left[-\frac{1}{2} \ln \left(1-Z^{2}\right)+Z \psi(Z)\right]\right|_{M} ^{Q(1)}-\int_{M}^{Q(1)} d Z \psi(Z) \\
& =\ln \cosh \dot{e}(M, 1)+\frac{1}{2} \ln \left(1-M^{2}\right)-M \psi(M)-E[Q(1)]+E(M)
\end{aligned}
$$

Using now the explicit expressions (4) and (17) for $e^{*}(m)$, we immediately obtain

$$
-\beta F=\ln 2+\mathrm{C}(\mathrm{M})+\mathrm{E}[\mathrm{Q}(1)]
$$

The behavior of $C(M)$ is thus completely determined by $Q(1)=$ $\tanh \dot{e}(M, s=\infty)$. The quantity $C(M)$ remains constant as long as $M$ is varied inside a range of values which have the same limit for $\tanh \dot{e}(M, s)$ as $s$ goes to infinity. Due to (56), the possible limiting values for this function are given by the roots of $\psi$, i.e., the roots $m_{i}$ of the equation

$$
m=\tanh \phi(m)
$$

A straightforward but important property of the solutions $\dot{e}(m, s)$ of our flow equation (15) is that the roots $m_{i}(s)$ of the equation

$$
m=\tanh \dot{e}(m, s)
$$

are constant with respect to $s$; they are thus equal to the roots $m_{i}$ of Eq. (59), and no new roots appear.

Since for finite $s$ the function $\dot{e}(m, s)$ is expected to remain continuous with respect to $m$, the discontinuities in the step function $\dot{e}(m, \infty)$ are expected to occur at $m$ values where $\partial \dot{e} / \partial m$ goes to infinity when $s$ becomes infinite. According to (15), this infinite slope can only be found for the values $m_{i}$ that obey (60).

Not all these values $m_{i}$, however, give rise to a discontinuity in $\dot{e}(m, \infty)$. From (15) we see that $\partial \dot{e} / \partial s$ changes its sign at the $m_{i}$. This means that, for growing $s$, the function $\dot{e}(m, s)$ becomes either more flat or more steep at these points, depending on the sign of $(m-\tanh \dot{e}) \partial \dot{e} / \partial m$. Let us clarify this by a typical situation in Fig. 5, where we have sketched $\psi(m)$ with, e.g., three roots, and the corresponding curve $\tanh \phi(m)=\tanh \dot{e}(m, 0)$. This last curve intersects the $m$ line at these three roots of $\psi$. In every interval we can look for the sign of $\partial \dot{e} / \partial s$, and we have indicated the corresponding renormalization flow of the function $\tanh \dot{e}(m, s)$ by arrows in Fig. 5. From this it can be seen that a discontinuity will only appear at the root $m_{2}$, where $\psi^{\prime}(m)$ is positive, and, as a consequence, $E(m)$ has a relative minimum. 


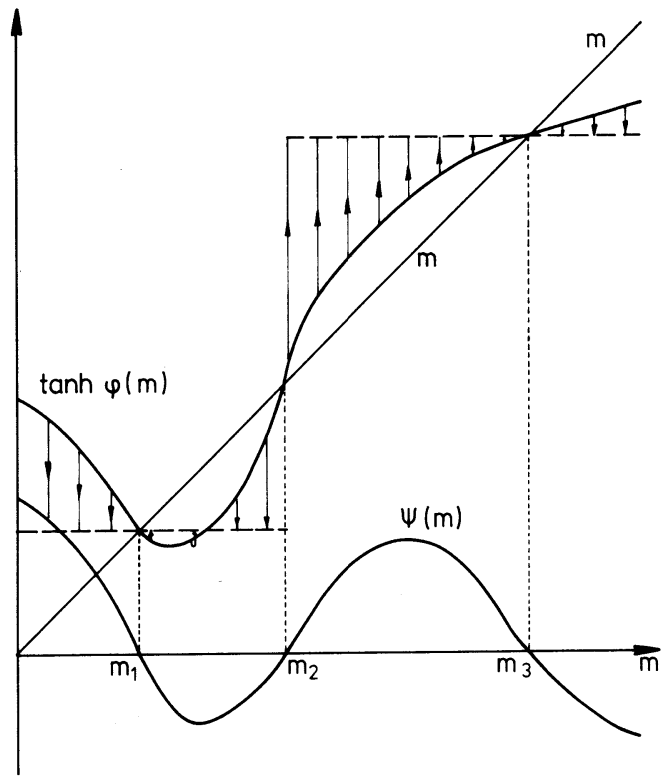

Fig. 5. Schematic representation of the renormalization flow of the function $\tanh \dot{e}(m, s)$. The roots of $\psi(m)$ are $m_{1}, m_{2}$, and $m_{3}$, and for these values of $m$ we have $m=\tanh \phi(m)=$ $\tanh \dot{e}(m, s)$ for all $s$. The step function indicated by the broken line and the isolated point at $\left(m_{2}, m_{2}\right)$ is the limit function $\tanh \dot{e}(m, \infty)$.

One can easily verify the generality of this result. The $Q(1)$ and, according to (58), also $C(M)$ are step functions of $M$ with discontinuities at the values of $M$ that correspond to the minima of $E(M)$. Comparing furthermore (58) with the exact result (39), we find that there is a whole range of $M$ values for which $C(M)=0$ and for which the integral expression in (25) gives the full free energy of the system. This range of $M$ values is the interval between two neighboring minima of $E(m)$, which contains the absolute maximum of this function.

Although the step function $\dot{e}(m, \infty)$ is a fixed-point solution of Eq. (15) for $\dot{e}(m, s)$, its integral $e(m, s)$ is not a fixed-point solution of Eq. (14). A function $e(m)$ with a piecewise constant derivative does not satisfy (14), unless we insert discontinuous jumps, proportional to $e^{s}$, at the points of discontinuity of the derivative. These jumps in $e(m, s \rightarrow \infty)$ are caused by divergences which build up in $\dot{e}(m, s)$ in a very narrow region around the discontinuities and give rise to a delta-function contribution to the limit $s \rightarrow \infty$. (For simplicity, we did not try to represent these divergences in Fig. 5.) An asymptotic solution $e(m, s)$ for large $s$ values is then formed by pieces of constant slope, which are moving with respect to one another either in the direction of positive or in the direction of negative energy values. 
One section, however, is fixed by our normalization condition $e(M, s)=$ $e(M, 0)$. The free energy $F(s)$, defined in (23), will then also be an exponentially growing function of $s$, unless all the moving sections of $e(m, s)$ are shifting toward negative values. It is clear that this will depend on the choice of the normalization point $M$.

\section{TRANSFORMATION WITH MODIFIED SPIN VARIABLES}

Up to now, in the discussion of our renormalization approach, we have put emphasis almost exclusively on the direct computation of the free energy as an integral along the renormalization trajectory. The conventional technique is to describe the critical properties of the system by analyzing the transformation in the proximity of the fixed point, without aiming at a global solution for the thermodynamic functions. In this section we show that this kind of analysis is also feasible within our infinitesimal approach.

Our method will be based on a simple infinitesimal "equivalence" transformation, which, combined with the decimation procedure of Section 2, allows us to construct a parameter-dependent class of infinitesimal transformations. This class can be viewed as an infinitesimal generalization of the transformations described by Knops et al. ${ }^{(4)}$

Let us first introduce a simple free-energy-conserving transformation of our spin variables, which does not reduce the number of degrees of freedom. We will use a set of new spin variables $S_{j}^{\prime}(j=1, \ldots, N)$ and we will link them to the old ones by means of weight functions $t\left(S_{j}, S_{j}{ }^{\prime}\right)$ according to the transformation scheme

$$
\begin{aligned}
\operatorname{Tr}_{\{S\}} \exp [N e(m)] & =\operatorname{Tr}_{\left\{S^{\prime}\right\}} \operatorname{Tr}_{\{S\}} \prod_{j}\left[t\left(S_{j}, S_{j}^{\prime}\right)\right] \exp [N e(m)] \\
& \equiv \operatorname{Tr}_{\left\{S^{\prime}\right\}} \exp \left[N \tilde{e}\left(m^{\prime}\right)+\tilde{g}\right]
\end{aligned}
$$

with $t$ obeying the normalization condition

$$
\operatorname{Tr}_{S^{\prime}} t\left(S, S^{\prime}\right)=1
$$

and $m^{\prime}$ defined as

$$
m^{\prime}=N^{-1} \sum_{j} S_{j}^{\prime}
$$

Since both $S$ and $S^{\prime}$ can take only the values \pm 1 , the only possibility is

$$
t\left(S, S^{\prime}\right)=\frac{1}{2}\left(1+p S S^{\prime}\right)
$$

When $p=1$, this amounts to an identity transformation $t\left(S, S^{\prime}\right)=\delta_{S S^{\prime}}$. 
We will choose $p$ to be infinitesimally different from unity, i.e., by an amount of order $N^{-1}$ :

$$
p=1+\alpha / N, \quad \alpha=O(1)
$$

The physical idea behind this transformation is to rescale the average spin values. The transformation of Section 2 preserves the thermal average of the spins, since $\langle m\rangle=\left\langle m^{\prime}\right\rangle$ after Eq. (9), as one can easily verify. The transformation that we now introduced, however, yields

$$
\left\langle m^{\prime}\right\rangle=p\langle m\rangle
$$

The thermal averages on the right- and left-hand sides of this equation have to be performed with the original and transformed Hamiltonians, respectively.

Both $\tilde{e}$ and $\tilde{g}$ are defined by (61), if we again choose some normalization, e.g., $\tilde{e}(0)=e(0)$ as in Section 2. Equation (61) is equivalent to

$$
\exp \left[\tilde{g}+N \tilde{e}\left(m^{\prime}\right)\right]=\operatorname{Tr}_{\{S\}} \exp [N e(m)] \prod_{j}\left(\delta_{S_{j}, S_{j}^{\prime}}+\frac{\alpha}{2 N} S_{j} S_{j}^{\prime}\right)
$$

By working out the product in the right-hand side, one gets a large sum of different terms. Each of these terms may be written, after renumbering of the indices, as

$$
\left(\frac{\alpha}{2 N}\right)^{k} \operatorname{Tr}_{\{S\}} \prod_{j=1}^{k}\left(S_{j} S_{j}^{\prime}\right) \prod_{j=k+1}^{N}\left(\delta_{S_{j} S_{j}}\right) e^{N e(m)}
$$

where $k$ is some positive integer. Neglecting terms of order $N^{-1}$ in the exponential, this term is equal to

$$
\begin{gathered}
\left(\frac{\alpha}{2 N}\right)^{k} \exp \left[N e\left(m^{\prime}\right)\right] \prod_{j=1}^{k}\left\{S_{j}^{\prime} \exp \left[-S_{j}^{\prime} \dot{e}\left(m^{\prime}\right)\right] \sum_{S= \pm 1} S \exp \left[S \dot{e}\left(m^{\prime}\right)\right]\right\} \\
=\exp \left[N e\left(m^{\prime}\right)\right] \prod_{j=1}^{k}\left[\frac{\alpha}{2 N}\left(S_{j}^{\prime}-\tanh \dot{e}\right) \sinh 2 \dot{e}\right]
\end{gathered}
$$

By repeating this for all terms, we finally have

$$
\begin{aligned}
\exp \left[\tilde{g}+N \tilde{e}\left(m^{\prime}\right)\right] & =\exp \left[N e\left(m^{\prime}\right)\right] \prod_{j=1}^{N}\left[1+\frac{\alpha}{2 N}\left(S_{j}^{\prime}-\tanh \dot{e}\right) \sinh 2 \dot{e}\right] \\
& \simeq \exp \left[N e\left(m^{\prime}\right)+\frac{1}{2} \alpha\left(m^{\prime}-\tanh \dot{e}\right) \sinh 2 \dot{e}\right]
\end{aligned}
$$

We will now combine the infinitesimal transformation of Section 2 with this rescaling of the spin variables. The combination of the two transformations leads in a straightforward way to an $\alpha$-dependent partial differential equation

$$
\frac{\partial \dot{e}}{\partial s}=\frac{\alpha}{2} \sinh 2 \dot{e}+\frac{\partial \dot{e}}{\partial m}[\tanh \dot{e}-m+\alpha(\cosh 2 \dot{e})(m-\tanh 2 \dot{e})]
$$


and a new $g(e)$, given by

$$
g=\ln 2 \cosh \dot{e}(0)-\alpha \sinh ^{2} \dot{e}(0)
$$

The fixed-point solution of (69) is the same as when $\alpha=0$, given by (16).

It is possible to construct the full solution of (69) with the initial condition $\dot{e}(m, s=0)=\phi(m)$ by the method of characteristic curves, much as we did for $\alpha=0$ in Section 3. This solution is given implicitly by

$$
\begin{aligned}
\tanh \dot{e}(m, s)= & e^{\alpha s} \tanh \phi\left\{e^{-\alpha s}\left[m e^{-s}+\left(1-e^{-s}\right) \tanh \dot{e}(m, s)\right]\right. \\
& \left.+2 e^{-s}(m-\tanh \dot{e}) \sinh \alpha s \cosh ^{2} \dot{e}(m, s)\right\}
\end{aligned}
$$

but in this form it is much more complicated to analyze than the solution (22) for $\alpha=0$. Instead, we will concentrate on a linearization around the fixed point.

If

$$
e(m, s)=e^{*}(m)+\sum_{n} a_{n}(s) m^{n}
$$

where, e.g., $a_{1}=h_{0}$, the magnetic field, and $a_{2}=\tau \sim T-T_{c}$, it is easy to show by a linearization of (69) or (71) in the $a_{n}$ that

$$
a_{n}(s) \simeq a_{n}(0) e^{(1-n \alpha) s}
$$

up to corrections of second order in the $a_{n}$. This result should be compared with (19), valid for $\alpha=0$. There all variables were relevant, but here it seems that the number of relevant and irrelevant variables can be chosen at will, by varying $\alpha$. A naive renormalization group analysis would thus seem to give $\alpha$-dependent critical exponents, since it now follows for the free energy that

$$
F(e)=F\left(\left\{a_{n}\right\}\right)=e^{-s} F\left(\left\{e^{(1-n \alpha) s} a_{n}\right\}\right)
$$

One way of solving this difficulty is to consider the free energy in (74) not only as a generalized homogeneous function of the $a_{n}$, described with a parameter $s$, but as a function whose properties are determined by two parameters $s$ and $\alpha$, or alternatively by $s$ and $\lambda=e^{-\alpha s}$. We may then interpret (74) or

$$
F\left(\left\{a_{n}\right\}\right)=e^{-s} F\left(\left\{e^{s} \lambda^{n} a_{n}\right\}\right)
$$

as a property that has to hold for all $s$ and all $\lambda$. This two-parameter freedom imposes much stronger conditions on $F$ than does the one-parameter condition (74) for a fixed $\alpha$.

As an application, let us investigate the consequences of this twoparameter condition on the situation where only three parameters are present: $a_{1}=h, a_{2}=\tau$, and $a_{4}$. We get

$$
F\left(h, \tau, a_{4}\right)=e^{-s} F\left(\lambda e^{s} h, \lambda^{2} e^{s} \tau, \lambda^{4} e^{s} a_{4}\right)
$$


Since this must hold for all $\lambda, F$ can only be a function of certain combinations of its three parameters; and it may be written as a new function of two independent combinations, e.g.,

$$
F\left(h, \tau, a_{4}\right)=G\left(h^{2} / \tau, \tau^{2} / a_{4}\right)=e^{-s} G\left(e^{s} h^{2} / \tau, e^{s} \tau^{2} / a_{4}\right)
$$

Since this must hold for all $s, G(x, y)$ must be a homogeneous function of the first degree in $x$ and $y$. For $h=0$ this means

$$
F\left(0, \tau, a_{4}\right) \sim \tau^{2} / a_{4}
$$

This eventual singular dependence on $a_{4}$ was described in Ref. 4 as the appearance of a dangerous irrelevant variable. If we ask $F$ to be finite for $\tau=0$ and $h \neq 0$, this implies from (76) that

$$
F\left(h, 0, a_{4}\right) \sim\left(h^{4} / a_{4}\right)^{1 / 3}
$$

The critical exponents may then be determined. For example, from (77) and (78), it follows immediately that $\alpha=0, \delta=3$. An analogous analysis for $\partial F / \partial h$ and $\partial^{2} \mathrm{~F} / \partial \mathrm{h}^{2}$ at $h=0$ gives $\beta=1 / 2$ and $\gamma=1$.

\section{CONCLUSIONS}

We have shown in this paper that it is possible to derive the full thermodynamic behavior of a van der Waals spin model by solving the differential renormalization equation obtained after summing out a single spin variable. A crucial problem was the determination of the integration constant $C$ appearing in the full solution (25) or (48) for the free energy equation. We have been able to show that in all cases, at least for a certain set of normalizations on the transformed Hamiltonians, this constant takes the value zero. One could hope that also in other situations, like those considered in Refs. 11 and 12, the troublesome constant may be eliminated by imposing conditions analogous to (46).

The nature of the fixed point, in our simplest renormalization procedure, is very peculiar, since all variables are relevant. We have modified this procedure şo as to keep only a few relevant variables. The usual linearization analysis in the neighborhood of the fixed point can then be performed. We have shown how the extra freedom of the transformation, manifested by the presence of a free parameter $\alpha$, leads to a strong limitation on the possible functional form of the free energy.

Since the method presented here is very simple, it is rather flexible and one can easily adapt it to treat, e.g., van der Waals model with higher dimensional spin variables, both in the classical and quantum cases. Generalizing the above scheme to the case in which the forces are not strictly infinitely long-ranged is a completely open problem. We are 
investigating for the moment the promising direction of imposing stochastic Glauber dynamics on our system; one may then try to perform a dynamic renormalization calculation, starting from the static scheme that was discussed here.

\section{REFERENCES}

1. K. G. Wilson and J. Kogut, Phys. Rep. 12:75 (1974).

2. 'Th. Niemeijer and J. M. J. van Leeuwen, in Phase Transitions and Critical Phenomena, C. Domb and M. S. Green, eds. (Academic Press, London, 1976), Vol. 6, p. 425.

3. C. Kittel and H. Shore, Phys. Rev. 138A:1165 (1965).

4. H. J. F. Knops, J. M. J. van Leeuwen, and P. C. Hemmer, J. Stat. Phys. 17:197 (1977).

5. R. S. Ellis and C. M. Newman, in Mathematical Problems in Theoretical Physics, G. Dell'Antonio, S. Doplicher, and G. Jona-Lasinio, eds. (Springer, Berlin, 1978), p. 313.

6. H. J. Hilhorst, M. Schick, and J. M. J. van Leeuwen, Phys. Rev. Lett. 40:1605 (1978); Phys. Rev. B 19:2749 (1979).

7. H. J. F. Knops and H. J. Hilhorst, Phys. Rev. B 19:3689 (1979).

8. Y. Yamazaki and H. J. Hilhorst, Phys. Lett. 70A:329 (1979).

9. W. van Saarloos, J. M. J. van Leeuwen, and A. L. Stella, Physica 97A:319 (1979).

10. R. Courant and D. Hilbert, Methods of Mathematical Physics (Interscience, New York, 1962), Vol. 2.

11. M. Nauenberg and B. Nienhuis, Phys. Rev. Lett. 33:1598 (1974).

12. D. R. Nelson, Phys. Rev. B 11:3504 (1975). 